\title{
Optimisation Problems for the Determinant of a Sum of $3 \times 3$ Matrices
}

\author{
FINBARR HOLLAND
}

\begin{abstract}
Given a pair of positive definite $3 \times 3$ matrices $A, B$, the maximum and minimum values of $\operatorname{det}\left(U^{*} A U+\right.$ $\left.V^{*} B V\right)$ are determined when $U, V$ vary within the collection of unitary $3 \times 3$ matrices.
\end{abstract}

\section{INTRODUCTION}

Let $m, n$ be a pair of natural numbers. Suppose $A_{1}, A_{2}, \ldots, A_{n}$ are $m \times m$ Hermitian positive definite matrices. What are the maximum and minimum values of the expression

$$
\operatorname{det}\left(\sum_{i=1}^{n} U_{i}^{*} A_{i} U_{i}\right)
$$

as $U_{1}, U_{2}, \ldots, U_{n}$ range over the group $G_{m}$ of $m \times m$ unitary matrices? The case $m=2$ of this arose in the context of an interesting maximum-likelihood problem which is discussed in [3], and the minimum value was determined there when the given matrices were real and symmetric, and the $U \mathrm{~s}$ members of the subgroup of $G_{2}$ of orthogonal matrices.

In this note we address the above problem only in the case $m=3$, and resolve it when $n=2$. However, the methods used here don't appear to generalise to the case of general $m$, even when $n=2$. Accordingly, a different strategy has been devised to deal with this more general case, which will be the subject of another paper. However, at the time of writing, the general case of arbitrary $m, n$ remains open.

2000 Mathematics Subject Classification. Primary 15A45.

Key words and phrases. Positive definite matrices, unitary matrices, doublystochastic matrices, extreme points, rearrangement inequality. 


\section{Statement of the Main Result}

Theorem 1. Let $S$ and $T$ be two $3 \times 3$ positive definite matrices with spectra $\sigma(S)=\left\{s_{1}, s_{2}, s_{3}\right\}$ and $\sigma(T)=\left\{t_{1}, t_{2}, t_{3}\right\}$, respectively, where $s_{1} \geq s_{2} \geq s_{3}>0$ and $t_{1} \geq t_{2} \geq t_{3}>0$. Then

$$
\min \left\{\operatorname{det}\left(S+U^{*} T U\right): U \in G_{3}\right\}=\prod_{i=1}^{3}\left(s_{i}+t_{i}\right),
$$

and

$$
\max \left\{\operatorname{det}\left(S+U^{*} T U\right): U \in G_{3}\right\}=\prod_{i=1}^{3}\left(s_{i}+t_{4-i}\right) .
$$

\section{Two Preparatory Lemmas}

Lemma 1. Let $A=\left[a_{i j}\right]$ be $a 3 \times 3$ matrix. Let

$$
M=\left[\begin{array}{ccc}
x+a_{11} & a_{12} & a_{13} \\
a_{21} & y+a_{22} & a_{23} \\
a_{31} & a_{32} & z+a_{33}
\end{array}\right] .
$$

Then

$\operatorname{det} M=x y z+y z a_{11}+z x a_{22}+x y a_{33}+x A_{11}+y A_{22}+z A_{33}+\operatorname{det} A$.

Proof. Here and later, we use the customary notation $A_{i j}$ for the cofactor of the typical element $a_{i j}$, so that, in particular, $A_{11}, A_{22}, A_{33}$ are the principal minors of $A$ of order $2 \times 2$. Expanding by elements of the first row,

$$
\begin{aligned}
\operatorname{det} M= & \left(x+a_{11}\right)\left[\left(y+a_{22}\right)\left(z+a_{33}\right)-a_{23} a_{32}\right] \\
& -a_{12}\left[a_{21}\left(z+a_{33}\right)-a_{31} a_{23}\right]+a_{13}\left[a_{21} a_{32}-a_{31}\left(y+a_{22}\right)\right] \\
= & \left(x+a_{11}\right)\left(y+a_{22}\right)\left(z+a_{33}\right)-\left[x a_{23} a_{32}+y a_{13} a_{31}+z a_{12} a_{21}\right] \\
& -a_{11} a_{23} a_{32}-a_{12}\left[a_{21} a_{33}-a_{31} a_{23}\right]+a_{13}\left[a_{21} a_{32}-a_{31} a_{22}\right] \\
= & \left(x+a_{11}\right)\left(y+a_{22}\right)\left(z+a_{33}\right)-a_{11} a_{23} a_{32} \\
& -\left[x a_{23} a_{32}+y a_{13} a_{31}+z a_{12} a_{21}\right]-a_{12} A_{12}+a_{13} A_{13} \\
= & x y z+x y a_{33}+y z a_{22}+z x a_{11}+x\left[a_{22} a_{33}-a_{23} a_{32}\right] \\
& +y\left[a_{11} a_{33}-a_{13} a_{31}\right]+z\left[a_{11} a_{22}-a_{12} a_{21}\right] \\
& \quad+a_{11} A_{11}-a_{12} A_{12}+a_{13} A_{13} \\
= & x y z+x y a_{33}+y z a_{22}+z x a_{11}+x A_{11}+y A_{22}+z A_{33}+\operatorname{det} A .
\end{aligned}
$$


We wish to exploit this result when $A=U^{*} T U$, where $T$ is a diagonal matrix with positive diagonal elements $p \geq q \geq r>0$, and $U=\left[u_{i j}\right]$ is unitary. A calculation shows that

$$
a_{i j}=p \overline{u_{i 1}} u_{j 1}+q \overline{u_{i 2}} u_{j 2}+r \overline{u_{i 3}} u_{j 3}, i, j=1,2,3 .
$$

In particular,

$$
a_{i i}=p\left|u_{i 1}\right|^{2}+q\left|u_{i 2}\right|^{2}+r\left|u_{i 3}\right|^{2}, i=1,2,3 .
$$

In addition, $A$ is invertible and $A^{-1}=U^{*} T^{-1} U=(\operatorname{det} A)^{-1}\left[A_{i j}\right]^{t}$, whence

$$
\frac{A_{i i}}{p q r}=p^{-1}\left|u_{i 1}\right|^{2}+q^{-1}\left|u_{i 2}\right|^{2}+r^{-1}\left|u_{i 3}\right|^{2}, i=1,2,3,
$$

or

$$
A_{i i}=q r\left|u_{i 1}\right|^{2}+r p\left|u_{i 2}\right|^{2}+p q\left|u_{i 3}\right|^{2}, i=1,2,3 .
$$

Observe too that

$$
\sum_{i=1}^{3}\left|u_{i j}\right|^{2}=\sum_{j=1}^{3}\left|u_{i j}\right|^{2}=1, i, j=1,2,3,
$$

and so the matrix $\left[\left|u_{i j}\right|^{2}\right]$ is doubly-stochastic. With this in mind we prove a rearrangement inequality.

Lemma 2. Let $\left[p_{i j}\right]$ stand for an arbitrary $n \times n$ doubly-stochastic matrix. Let $a, b$ be two real $n \times 1$ vectors whose entries are in decreasing order. Then

$$
\sum_{i=1}^{n} a_{i} b_{n-i+1} \leq \sum_{i, j=1}^{n} a_{i} b_{j} p_{i j} \leq \sum_{i=1}^{n} a_{i} b_{i} .
$$

Proof. Consider the function $f$ defined on the convex set $\mathcal{P}$ of all $n \times n$ doubly-stochastic matrices $P=\left[p_{i j}\right]$ by

$$
f(P)=\sum_{i, j=1}^{n} a_{i} b_{j} p_{i j}, \quad P \in \mathcal{P} .
$$

Clearly, $f$ is linear in $P$, and so convex on $\mathcal{P}$. Hence it attains its maximum and minimum at an extreme point of $\mathcal{P}$. But, by Birkhoff's theorem [1], the set of extreme points of the latter consists of the set of permutation matrices $\left\{\pi(I)=\left[\delta_{i \pi(j)}\right]: \pi \in S_{n}\right\}$, where $S_{n}$ 
denotes the group of permutations of $\{1,2, \ldots, n\}$. Hence

$$
\begin{aligned}
\min \{f(P): P \in \mathcal{P}\} & =\min \left\{f(\pi(I)): \pi \in S_{n}\right\} \\
& =\min \left\{\sum_{i, j=1}^{n} a_{i} b_{j} \delta_{i \pi(j)}: \pi \in S_{n}\right\} \\
& =\min \left\{\sum_{j=1}^{n} a_{\pi(j)} b_{j}: \pi \in S_{n}\right\} \\
& =\sum_{j=1}^{n} a_{j} b_{n-j+1},
\end{aligned}
$$

by the elementary rearrangement inequality, since $a, b$ are similarly ordered [2]. This argument establishes that

$$
\sum_{i=1}^{n} a_{i} b_{n-i+1} \leq \sum_{i, j=1}^{n} a_{i} b_{j} p_{i j},
$$

with equality when $p_{i j}=\delta_{i(n-j+1)}, i, j=1,2, \ldots, n$,

The maximum can be handled in the same way.

\section{Proof of the Main Result}

Define $F$ on the group $G_{3}$ of $3 \times 3$ unitary matrices by

$$
F(U)=\operatorname{det}\left(S+U^{*} T U\right), U \in G_{3} .
$$

In the first place, there are matrices $V, W \in G_{3}$ such that

$$
S=V\left[\begin{array}{ccc}
s_{1} & 0 & 0 \\
0 & s_{2} & 0 \\
0 & 0 & s_{3}
\end{array}\right] V^{*} \equiv V \Delta V^{*},
$$

and

$$
T=W\left[\begin{array}{ccc}
t_{1} & 0 & 0 \\
0 & t_{2} & 0 \\
0 & 0 & t_{3}
\end{array}\right] W^{*} \equiv W \Lambda W^{*},
$$

say. Hence

$$
F\left(W U V^{*}\right)=\operatorname{det}\left(\Delta+U^{*} \Lambda U\right),
$$

whence it's enough to deal with the case where $S=\Delta, T=\Lambda$. This being so, we can appeal to Lemma 1, taking

$$
A=U^{*} \Delta U=\left[\sum_{k=1}^{3} t_{k} \overline{u_{i k}} u_{j k}\right],
$$


and obtain that

$$
\begin{aligned}
\operatorname{det}\left(\Delta+U^{*} \Lambda U\right)= & \operatorname{det}(\Delta+A) \\
= & s_{1} s_{2} s_{3}+s_{1} s_{2} s_{3} \sum_{k=1}^{3} s_{k}^{-1} a_{k k}+\sum_{k=1}^{3} s_{k} A_{k k}+\operatorname{det} A \\
= & s_{1} s_{2} s_{3}+s_{1} s_{2} s_{3} \sum_{i=1}^{3} s_{i}^{-1} \sum_{j=1}^{3} t_{j}\left|u_{i j}\right|^{2} \\
& \quad+t_{1} t_{2} t_{3} \sum_{i=1}^{3} s_{i} \sum_{j=1}^{3} t_{j}^{-1}\left|u_{i j}\right|^{2}+t_{1} t_{2} t_{3} \\
= & s_{1} s_{2} s_{3}+s_{1} s_{2} s_{3} \sum_{i, j=1}^{3} s_{i}^{-1} t_{j}\left|u_{i j}\right|^{2} \\
& \quad+t_{1} t_{2} t_{3} \sum_{i, j=1}^{3} s_{i} t_{j}^{-1}\left|u_{i j}\right|^{2}+t_{1} t_{2} t_{3} \\
& s_{1} s_{2} s_{3}+s_{1} s_{2} s_{3} \sum_{i=1}^{3} s_{i}^{-1} t_{i}+t_{1} t_{2} t_{3} \sum_{i=1}^{3} s_{i} t_{i}^{-1}+t_{1} t_{2} t_{3},
\end{aligned}
$$

by Lemma 2 , since $s_{1}, s_{2}, s_{3}$, and $t_{1}^{-1}, t_{2}^{-1}, t_{3}^{-1}$ are oppositely ordered.

It follows that

$$
\begin{aligned}
\operatorname{det}\left(\Delta+U^{*} \Lambda U\right) \geq & s_{1} s_{2} s_{3}+t_{1} s_{2} s_{3}+t_{2} s_{1} s_{3}+t_{3} s_{1} s_{2} \\
& +s_{1} t_{2} t_{2}+t_{2} s_{1} s_{3}+t_{3} s_{1} s_{2}+t_{1} t_{2} t_{3} \\
= & \left(s_{1}+t_{1}\right)\left(s_{2}+t_{2}\right)\left(s_{3}+t_{3}\right),
\end{aligned}
$$

with equality when $U=I$, the identity matrix. Hence

$$
\min \left\{F(U): U \in G_{3}\right\}=\prod_{i=1}^{3}\left(s_{i}+t_{i}\right) .
$$

Arguing in a similar manner, it can be seen that

$$
\max \left\{F(U): U \in G_{3}\right\}=\prod_{i=1}^{3}\left(s_{i}+t_{4-i}\right) .
$$

This completes the proof of Theorem 1 . 


\section{REFERENCES}

[1] R. Bhatia, Matrix Analysis, Springer-Verlag, 1997.

[2] G. H. Hardy, J. E. Littlewood And G. Pólya, Inequalities, Cambridge University Press, 1934.

[3] F. Holland and K. Roy Choudhrey, Likelihood ratio tests for equality of shape under varying degrees of orientation invariance, J. Multivariate Analysis 99 (2008), 1772-1792.

Finbarr Holland,

Mathematics Department,

University College,

Cork, Ireland,

f.holland@ucc.ie

Received on 25 July 2008. 